\title{
Distribuição espaço - temporal de Sclerotinia Sclerotiorum em campo de feijoeiro tratado com diferentes métodos de controle foliar
}

\author{
Iara Silva Rocha ${ }^{1}$, Jakelinny Martins Silva ${ }^{1}$, Anderson Rodrigo Silva ${ }^{1}$, Anderson Rodrigues Rietjens ${ }^{1}$, \\ Natanael Marcos Lemes ${ }^{1}$, Milton Luíz da Paz-Lima ${ }^{1}$
}

\begin{abstract}
${ }^{1}$ Instituto Federal Goiano Campus Urutaí - Rod. Geraldo Silva Nascimento, Km-2,5 - Zona Rural, Urutaí - GO, Brasil; RC Cruz Estação Experimental - Fazenda Esmeralda, rodovia BR 050, Km 41, latitude: 17²9’31.35”, longitude: 48¹2’56.93”, Ipameri, Goiás, Brasil.

Autor para correspondência: Anderson Rodrigues Rietjens (andersonrietjens1@gmail.com)

Data de chegada: 05/10/2016. Aceito para publicação em: 11/05/2017.
\end{abstract}

$10.1590 / 0100-5405 / 170227$

\section{RESUMO}

Rocha, I.S.; Silva, J.M.; Silva, A.R.; Rietjens, A.R.; Lemes, N.M.; Paz-Lima, M.L.. Distribuição espaço - temporal de Sclerotinia Sclerotiorum em campo de feijoeiro tratado com diferentes métodos de controle foliar. Summa Phytopathologica, v.44, n.4, p.361-367, 2018.

O objetivo deste trabalho foi caracterizar a distribuição espaço-temporal de Sclerotinia sclerotiorum em campo de feijoeiro tratado com diferentes métodos de controle foliar. O feijão cultivar Pérola foi cultivado em regime de Pivô Central [safra de inverno (seca), 2015] na Fazenda São José, Cristalina, Góias. Foram aplicados nove tipos de tratamentos representados por fungicidas e agentes de controle biológico, ministrados em quatro aplicações, conduzidas em seis blocos (DBC), totalizando 54 unidades experimentais. Na área experimental avaliou-se a distribuição espacial do inóculo infuenciado pelos tratamentos aos 39 dias após o plantio (DAP), 46 DAP, 53 DAP, 60 DAP, 67 DAP e 74 DAP.
Para este estudo avaliou-se a incidência do mofo-branco (\%IMB) nos quadrantes $\left(36 \mathrm{~m}^{2}\right)$, sendo calculado a área abaixo da curva de progresso da doença (AACPD) e a produtividade (P) aos 87 DAP. Os valores de \%IMB, AACPD e P foram organizados em matrizes $\mathrm{X}(\mathrm{m}), \mathrm{Y}(\mathrm{m})$ e $\mathrm{Z}(\%)$ para construção de mapas de distribuição. Tanto no ciclo vegetativo como reprodutivo da cultura, os focos de incidência da doença mudaram de posição espacial e aumentaram aleatoriamente a incidência miceliogênica e carpogênica na área avaliada. O emprego de medidas sanitárias de controle do mofo branco influencia na dinâmica de dispersão do mofo-branco do feijoeiro.

Palavras-chave: mofo-branco, dispersão espacial, incidência, ciclo miceliogênico, ciclo carpogênico.

\section{ABSTRACT}

Rocha, I.S.; Silva, J.M.; Silva, A.R.; Rietjens, A.R.; Lemes, N.M.; Paz-Lima, M.L.. Spatio-temporal distribution of Sclerotinia Sclerotiorum in a bean field treated with different leaf control methods. Summa Phytopathologica, v.44, n.4, p.361-367, 2018.

The objective of this study was to characterize the spatio-temporal distribution of Sclerotinia sclerotiorum in a bean field treated with different leaf control methods. The bean cultivar Pérola was grown in Central Pivot (winter crop season [dry], 2015) in the Farm São José, Cristalina, Goiás State, Brazil. Nine types of treatments represented by fungicides and biological control agents were used in four applications conducted in six blocks (RBD), totaling 54 experimental units. In the experimental area, the spatial distribution of the inoculum was evaluated under the influence of the treatments 39 days after planting (DAP), 46 DAP, 53 DAP, 60 DAP, 67 DAP and 74 DAP. For this study, the incidence of white mold (\% IWM) was evaluated in the quadrants $\left(36 \mathrm{~m}^{2}\right)$, and the area under the disease progress curve (AUDPC) was calculated, as well as productivity $(\mathrm{P})$ at $83 \mathrm{DAP}$. Values of $\%$ IWM, AUDPC and $\mathrm{P}$ were arranged in matrices $\mathrm{X}(\mathrm{m}), \mathrm{Y}(\mathrm{m})$ and $\mathrm{Z}(\%)$ for the elaboration of distribution maps. In both vegetative cycle and reproductive cycle of the crop, the disease incidence foci changed their spatial position and randomly increased the myceliogenic and carpogenic incidence in the evaluated area. The use of sanitary measures for white mold control influences the dynamic of the dispersion of white mold in bean plants.

Keywords: white mold, spatial dispersion, incidence, myceliogenic cycle, carpogenic cycle.

O feijoeiro comum (Phaseolus vulgaris L. - Fabaceae) é uma das culturas mais importantes do Brasil. Seu cultivo encontra-se associado a cultura de subsistência desenvolvido algumas vezes por pequenos produtores, por se tratar de uma cultura explorada comercialmente de maneira insipiente. No entanto, existem grandes produtores localizados em áreas de destaque no Brasil, onde aplica-se cultivos altamente mecanizados, como o uso de sistemas em pivô Central no Brasil Central. É também um dos alimentos básicos da população brasileira, destacando-se como fontes de proteínas, ferro e carboidratos (14). O agronegócio de feijão no Brasil, necessita de atenção dos produtores e pesquisadores visando a manutenção e aumento de produtividade nos campos de produção. E para manutenção de índices de rendimento competitivos, a redução dos efeitos do mofo branco (Sclerotinia sclerotiorum (Lib.) De Bary) permitem o desenvolvimento satisfatório do setor. $\mathrm{O}$ ambiente, representado por áreas de umidade relativa $\mathrm{e}$ temperatura do ar acima de $70 \%$ e $35^{\circ} \mathrm{C}$, respectivamente, favorecem a ocorrência de várias patologias (2) além do mofo branco, que diminuírem a produtividade e depreciam a qualidade do produto (11).

Para Ricardo et al. (10), a disseminação de doenças fúngicas, como o mofo-branco, é favorecida pelo avanço do cultivo irrigado, principalmente pela má qualidade de sementes, que introduzem o patógeno em novas áreas, e pelo ambiente altamente favorável ao desenvolvimento do mofo branco. Ghini et al. (6) apontaram que em temperaturas amenas e elevadas condições de umidade o patógeno é favorecido, enquanto, em condições adversas pode permanecer em restos de cultura e solo por mais de oito anos, na forma de escleródios que variam muito de tamanho, o hospedeiro e consequente expressão variável do ciclo da doença, uma vez que produzem o inóculo para os 
ciclos primário (sobrevivência) e ciclo secundário.

O agente causal $S$. sclerotiorum apresenta ciclo sexual, o que é importante na geração de variabilidade e no potencial evolutivo das populações de patógenos (4). O patógeno está difundido por todo o país, e as áreas mais afetadas são os estados do Sul, Sudeste e CentroOeste, locais com condições climáticas favoráveis ao patógeno, geralmente regiões acima de $800 \mathrm{~m}$ de altitude (1). Ricardo et al. (10) demonstraram que o mofo branco, representado por sua fase miceliogênica, no estado de GO, vem causando grandes prejuízos em lavouras de feijoeiro comum de $3^{\mathrm{a}}$ safra e que estes prejuízos representam perdas significativas para o Estado. Porém, essa redução na produtividade representa um dano econômico mais expressivo que os gastos com seu controle.

O relacionamento entre os padrões espaciais e o número de apotécios de S. sclerotiorum foi estudada por Boland \& Hall (3), afirmando que o mofo-branco (fase miceliogênica) e a produção de apotécios (fase carpogênica) possuem padrões de distribuição espacial agregada. Espacialmente foi identificado o número de 40 apotécios por $\mathrm{m}^{2}$ mensurado por Williams \& Stelfox (15), e a dispersão de ascósporos foi de $25 \mathrm{~m}$ a muitos $\mathrm{km}$ (13). Os estudos de distribuição espacial do mofo-branco em diferentes hospedeiras concentram-se em relacionar a distância com valores de incidência apenas para a fase carpogênica.

O objetivo deste trabalho foi realizar a caracterização e análise da distribuição espacial da fase sexual de $S$. sclerotiorum incidente em feijoeiro submetido a diferentes fungicidas e agentes de biocontrole.

\section{MATERIAL E MÉTODOS}

O experimento foi realizado em regime de pivô central, ano agrícola de 2015, situado na Fazenda São José, Cristalina, Góias. O cultivo foi realizado em sistema de plantio direto, utilizando-se a cultivar Pérola, sendo antecedido a cultura do feijão o plantio de milho. A adubação de plantio foi realizada com $270 \mathrm{~kg} \mathrm{ha}^{-1}$ do formulado $05-37-00 \mathrm{e}$ cloreto de potássio $\left(90 \mathrm{~kg} \mathrm{ha}^{-1}\right)$ sendo aplicado utilizando o sistema de agricultura de precisão.

A semeadura foi realizada em 30 de maio de 2015, e foram aplicados 9 tipos de tratamentos, ministrados em quatro aplicações, conduzidos em 6 blocos por tratamentos com fungicida, em delineamento em blocos casualizados, totalizando 54 unidades experimentais. Cada parcela apresentou dimensões de $6 \times 6 \mathrm{~m}$, com área de $36 \mathrm{~m}^{2}$ por parcela e espaçamento de entre linhas utilizado foi de $0,5 \mathrm{~m}$, enquanto entre plantas foi de $0,2 \mathrm{~m}$. Foram desprezados $0,5 \mathrm{~m}$ das extremidades das duas linhas centrais, totalizando uma área útil de $9 \mathrm{~m}^{2}$. As avaliações foram realizadas nas dez linhas centrais. O número total de plantas por linha foi de 30 plantas, totalizando 300 plantas por parcela.

Foram realizadas até quatro aplicações ( $1^{\mathrm{a}}$ aplicação aos 18 dias após o plantio (DAP); $2^{\mathrm{a}}$ aos 26 DAP; $3^{\mathrm{a}}$ aos 34 DAP e $4^{\mathrm{a}}$ aos 46 DAP). Os tratamentos foram representados por: T1 testemunha (sem aplicação), T2 Bacillus subtilis linhagem QST 713 - Serenade $^{\circledR}$ (4 $\mathrm{L} \mathrm{ha}^{-1}$ aplicado no estádio V3), T3 Bacillus subtilis linhagem QST 713 - Serenade ${ }^{\circledR}$ (4 L ha ${ }^{-1}$ aplicado no estádio V3 e V4), T4 Bacillus subtilis linhagem QST 713 - Serenade ${ }^{\circledR}\left(2 \mathrm{~L} \mathrm{ha}^{-1}\right.$ aplicado no estádio V3 e V4), T5 Bacillus subtilis linhagem QST 713 - Serenade $^{\circledR}$ e Trifloxistrobina + protioconazol - Fox ${ }^{\circledR}\left(4\right.$ e $0,5 \mathrm{~L} \mathrm{ha}^{-1}$, aplicados nos estádios, V3; V4, R5 e R5+10 dias), T6 Bacillus subtilis linhagem QST 713 - Serenade $^{\circledR}$ e Trifloxistrobina + protioconazol - Fox ${ }^{\circledR}(2$ e 0,5 $\mathrm{L} \mathrm{ha}^{-1}$, aplicados nos estádios V3; V4, R5 e R5+10 dias), T7 Bacillus subtilis linhagem QST 713 - Serenade ${ }^{\circledR}$, Trifloxistrobina
+ protioconazol - Fox ${ }^{\circledR}$ e Fluazinam - Frowncide ${ }^{\circledR}\left(2 ; 0,5\right.$ e 1 L ha $^{-1}$, aplicados nos estádios V3, V4; V4, R5, R5+10 dias; V4 e R5) e T8 Trifloxistrobina + protioconazol - Fox ${ }^{\circledR}$ e Fluazinam - Frowncide ${ }^{\circledR}(0,5$ e $1 \mathrm{~L} \mathrm{ha}^{-1}$, aplicados os estádios V4, R5, R5+10dias; V4 e R5) e T9 Trichoderma harzianum Esalq 1306 / Trichodermil ${ }^{\circledR}$, Azoxistrobina + Difenoconazol - Amistar Top ${ }^{\circledR}$ e Fluazinam - Frowncide ${ }^{\circledR}(1,5 ; 0,5$ e 1 $\mathrm{L} \mathrm{ha}^{-1}$, V3, V4; V4, R5, R5+10dias; V4 e R5).

A fase sexuada foi quantificada em cada parcela pela observação de sua presença na superfície do solo das estruturas sexuais denominadas de apotécios. A fase assexuada ou incidência da doença (\%) foi avaliada aos 39 dias após o plantio (DAP), 46 DAP, 53 DAP, 60 DAP, 67 DAP e 74 DAP, sendo tomadas cinco medidas representadas pelo número de plantas sintomáticas (sintoma de mofo-branco) dividido pelo número total de plantas (10 plantas analisadas). A produtividade das parcelas foi avaliado aos 87 DAP (dessecação 2 dias antes da colheita, apresentando $70 \%$ das folhas secas).

O centro de cada parcela experimental de dimensões 6 × $6 \mathrm{~m}$ foi referenciado no sistema de coordenadas cartesianas, formando um grid regular. A terceira dimensão (coordenada $\mathrm{z}$ ) representou a incidência do mofo-branco (\%), aos 39 dias após o plantio (DAP), 46 DAP, 53 DAP, 60 DAP, 67 DAP e 74 DAP, e a produtividade aos 87 DAP.

Utilizou-se o programa Statistica ${ }^{\circledR}$ adicionando as três coordenadas, utilizando o procedimento GRAPH “contour plots" para construção de gráficos em 2D.

A apresentação gráfica em todos os dias de avaliação foi organizada para demonstrar o efeito espacial e a dispersão do patógeno sob ótica temporal em diferentes estádios de desenvolvimento da cultura.

Utilizando o programa R e o pacote Biotools (12) a partir das matrizes $\mathrm{X}, \mathrm{Y}$ e $\mathrm{Z}$, onde $\mathrm{Z}$ era representado pelas incidências aos 46 DAP, 53 DAP, 60 DAP, 67 DAP e 74 DAP, aplicou-se o teste de correlação de Mantel (realiza um teste de hipótese de correlação entre as distâncias geográficas e a incidência do mofo branco) para relacionar a distância geográfica com as incidências do mofo branco. Os mapas de distribuição sem o efeito dos tratamentos foi realizado através das matrizes de resíduos (8). Criou-se mapas de distribuição espacial (Fig. 2 e 3) para os valores de incidência, AACPD e produtividade, bem como, calculou-se as áreas de ocupação geográfica das mesmas, submetidas a testes de hipótese (Tab. 1 e 2).

\section{RESULTADOS E DISCUSSÃO}

Distribuição da fase assexuada sexuada ou carpogênica em área comercial de cultivo de feijão comum tratado com diferentes fontes de fungicidas químicos e agentes de biocontrole.

Nas avaliações 39 e 46 DAP (Fig. 1AB) não foram detectados focos da distribuição espacial da fase carpogênica de S. sclerotiorum. Somente a partir dos 53 DAP (Fig. 1C) pode-se detectar a presença de apotécios na parcela coincidentemente que não havia sido aplicado tratamentos para controle (Testemunha). Aos 60 DAP (Fig. 1D) foi detectado 3 focos da produção de apotécios, sendo detectado nas parcelas controle e em duas parcelas onde se efetuou o tratamento T6 Bacillus subtilis linhagem QST 713 - Serenade ${ }^{\mathbb{R}}$ e Trifloxistrobina + protioconazol Fox $^{\circledR}$ (2 e 0,5 L ha ${ }^{-1}$, aplicados nos estádios V3; V4, R5 e R5+10 dias). Aos 67 DAP (Fig. 1E) foram detectados 18 focos da fase carpogênica nas parcelas representadas pelos tratamentos T1 (1 foco), T2 (2 focos), T3 (2 focos), T4 (1 foco), T5 (1 foco), T6 (2 focos), T7 (2 focos), T8 ( 3 focos) e T9 ( 2 focos). Aos 74 DAP não foi detectado a presença de apotécios nas parcelas experimentais, sendo importante ressaltar que 
devido a aproximação da data de colheita, houve restrição hídrica da lavoura, e possivelmente tenha influenciado o reconhecimento e a identificação da fase apotecial de $S$. sclerotiorum. Nos demais dias de avaliação a irrigação no sistema de pivô foi fornecida diariamente sem restrição ou controle de lâmina d'água na lavoura (Fig. 1).

Focos do inóculo são apontados (Fig. 1E) e distribuídos em diversos tratamentos, sendo os mais frequentes no Tratamento 6 $\left(\right.$ Serenade $^{\circledR}$ e Fox $^{\circledR}$ ), Tratamento $8\left(\right.$ Fox $^{\circledR}$ e Frowncide ${ }^{\circledR}$ ) e Tratamento 9 (Trichodermil $^{\circledR}$, Amistar Top ${ }^{\circledR}$ e Frowncide ${ }^{\circledR}$ ). Diferente dos demais dias avaliados, na sexta avaliação não houve detecção perceptível da presença de apotécios.

Interpolação da distribuição de inóculo da fase miceliogênica ou assexual em área comercial de cultivo de feijão comum tratado com diferentes fontes de fungicidas químicos e agentes de biocontrole.

Aos 39 DAP pode-se observar a presença de dois focos marginais de incidência da doença (incidência máxima $=2 \%$ )(Fig. 2A). Aos 46 DAP observaram-se dois focos marginais e um central, sendo a porcentagem máxima de incidência do mofo-branco encontrado na área de 10 \% (Fig. 2B). Aos 60 DAP observou-se a presença de 4 focos marginais e um central, sendo a incidência máxima do mofo-branco na área de $30 \%$ (Fig. 2C). Aos 67 DAP observou-se a presença de 3 focos marginais, sendo a incidência máxima atingida de $40 \%$ (Fig. 2D). Nesses quatro dias de avaliação a incidência da doença na área elevouse. Até esta última avaliação (67 DAP) o regime de irrigação diário realizou-se sem controle da quantidade aplicada na lavoura (influência no progresso da doença), iniciando então um regime de restrição hídrica para a realização da colheita, influenciando na Figura 2E a redução da incidência e o número de focos na área avaliada.

A incidência e a distribuição do mofo-branco na área foi influenciada pela aplicação de fungicidas químicos e agentes biológicos. No entanto, isto não expressou maior quantidade de inóculo nos tratamentos controle (sem aplicação de agentes de controle), pois a partir dos 67 DAP ficou provado que a incidência da fase carpogênica e esporogênica não foi conduzida ou controlada pelos agentes de manejo foliar aplicado nos quadrantes (Fig. 1 e 2).

A severidade do mofo-branco causado por S. sclerotiorum em diferentes hospedeiros é proporcional à densidade de inóculo presente no solo. Portanto, a redução da população de escleródios é essencial para o controle efetivo do mofo-branco no solo, que, além disso, também
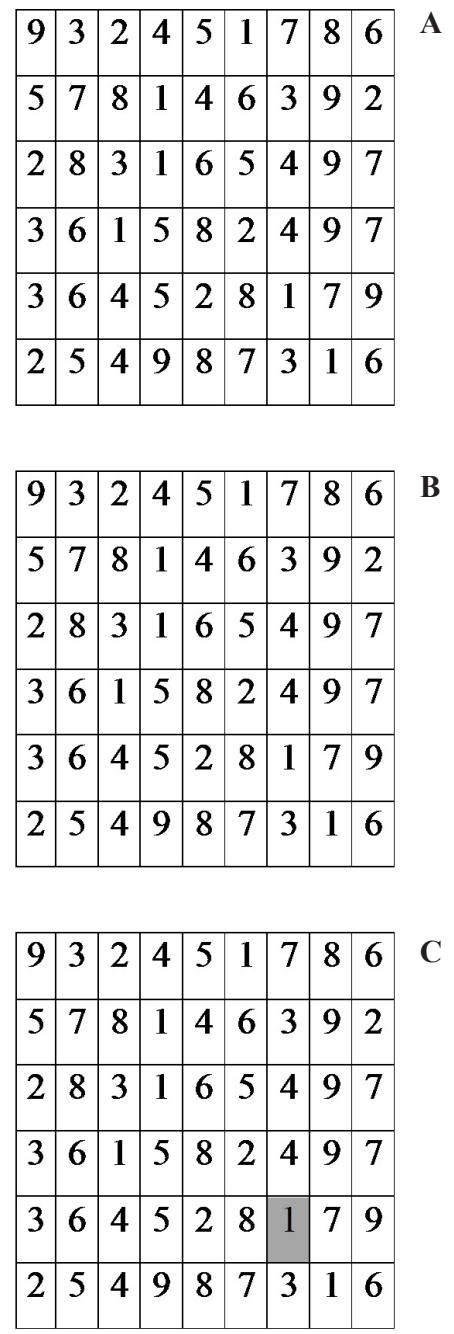

\begin{tabular}{|l|l|l|l|l|l|l|l|l|}
\hline 9 & 3 & 2 & 4 & 5 & 1 & 7 & 8 & 6 \\
\hline 5 & 7 & 8 & 1 & 4 & 6 & 3 & 9 & 2 \\
\hline 2 & 8 & 3 & 1 & 6 & 5 & 4 & 9 & 7 \\
\hline 3 & 6 & 1 & 5 & 8 & 2 & 4 & 9 & 7 \\
\hline 3 & 6 & 4 & 5 & 2 & 8 & 1 & 7 & 9 \\
\hline 2 & 5 & 4 & 9 & 8 & 7 & 3 & 1 & 6 \\
\hline
\end{tabular}

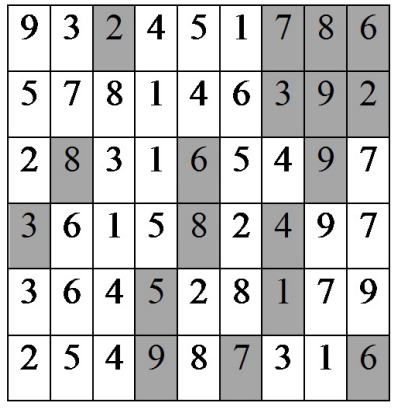

\begin{tabular}{|l|l|l|l|l|l|l|l|l|}
\hline 9 & 3 & 2 & 4 & 5 & 1 & 7 & 8 & 6 \\
\hline 5 & 7 & 8 & 1 & 4 & 6 & 3 & 9 & 2 \\
\hline 2 & 8 & 3 & 1 & 6 & 5 & 4 & 9 & 7 \\
\hline 3 & 6 & 1 & 5 & 8 & 2 & 4 & 9 & 7 \\
\hline 3 & 6 & 4 & 5 & 2 & 8 & 1 & 7 & 9 \\
\hline 2 & 5 & 4 & 9 & 8 & 7 & 3 & 1 & 6 \\
\hline
\end{tabular}

$\mathbf{E}$

\section{D}

F

Figura 1. Detecção e distribuição espacial da fase carpogênica (presença marcados em cinza) de Sclerotinia sclerotiorum nos blocos tratados com diferentes combinações, dosagens e épocas de aplicação de fungicidas avaliados aos 39 dias após o plantio DA (DAP) (A), 46 DAP (B), 53 DAP (C), 60 DAP (D), 67 DAP (E) e 74 DAP (F). 
deve bloquear a formação de apotécios e a liberação de ascósporos. Minimizando a produção de novos escleródios pelo controle preventivo da doença na parte aérea das plantas (7).

Análise espacial do inóculo de Sclerotinia sclerotiorum em área comercial de cultivo de feijão comum tratado com diferentes fontes de fungicidas químicos e agentes de biocontrole.

Quanto maiores seus valores de AACPD, maior a quantidade do mofo-branco desenvolvido na parcela. Sem o efeito dos tratamentos observou-se $12+6$ focos externos+internos no mapa de distribuição espacial da AACPD (Fig. 3A), respectivamente; já com efeito dos tratamentos observou-se $11+6$ focos externos + internos no mapa de distribuição espacial da AACPD (Fig. 3B). O inóculo inicial (Q0) representado pelo número de focos, foi responsável pelo progresso aleatório da doença. A redução da produtividade da cultura não foi observada tanto analisando esse inóculo com ou sem efeito dos tratamentos aéreos ao final do experimento.
Já quando falamos da distribuição espacial da produtividade (Fig. $3 \mathrm{CD}$ ), sem o efeito dos tratamentos, observamos uma variação da produtividade, sendo verificado 1 foco marginal que representou a maior produtividade da cultura $\left(1800 \mathrm{~kg} \mathrm{ha}^{-1}\right)$, valendo lembrar que CONAB (5) aponta que a média nacional de produtividade na 2015 safra de inverno foi de $1000 \mathrm{~kg} \mathrm{ha}^{-1}$, e ainda afirma que produtores altamente tecnificados chegam a patamares de $2000 \mathrm{~kg} \mathrm{ha}^{-1}$. Assim, agronomicamente é importante verificar o manejo local para expressálo em toda área avaliada. Considerando o mapa de distribuição da produtividade sob efeito dos tratamentos, observou-se apenas um único foco marginal que estabelece a variação dos patamares de produtividade para 400 a $1800 \mathrm{~kg} \mathrm{ha}^{-1}$, sendo que nas áreas de 1600-1800 a influência dos tratamentos T5 Bacillus subtilis linhagem QST 713 - Serenade ${ }^{\circledR}$ e Trifloxistrobina + protioconazol - Fox ${ }^{\circledR}\left(4\right.$ e $0,5 \mathrm{~L} \mathrm{ha}^{-1}$, aplicados nos estádios, V3; V4, R5 e R5+10 dias) e T9 Trichoderma harzianum Esalq 1306 Trichodermil $^{\mathbb{B}}$, Azoxistrobina + Difenoconazol - Amistar Top $^{\circledR}$ e Fluazinam - Frowncide ${ }^{\circledR}\left(1,5 ; 0,5\right.$ e $1 \mathrm{~L} \mathrm{ha}^{-1}$, V3, V4; V4, R5,
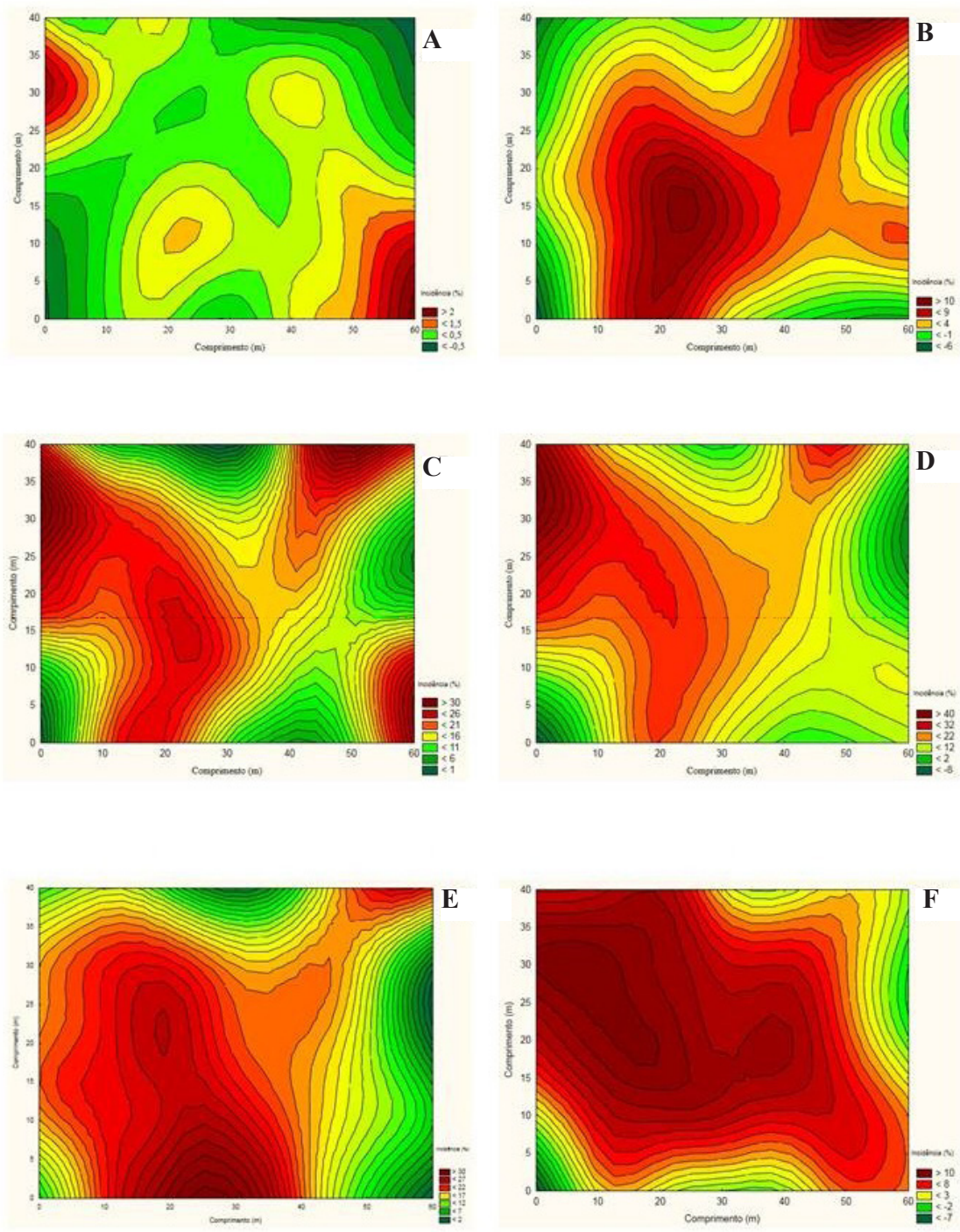

Figura 2. Interpolações da distribuição espacial da fase miceliogênica de Sclerotinia sclerotiorum em diferentes blocos de feijoeiro-comum tratados com fungicidas e agentes de controle biológico. A- distribuição aos 39 DAP, B - distribuição aos 46DAP, C - distribuição aos 53DAP, D- distribuição aos 60 DAP, E- distribuição aos 67 DAP e F- distribuição aos 74 DAP. 


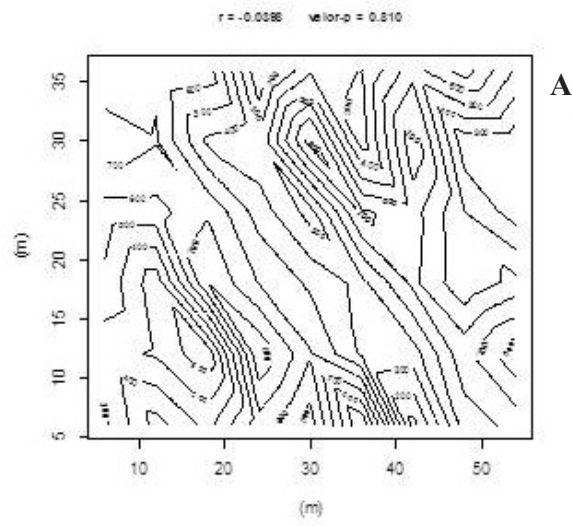

A

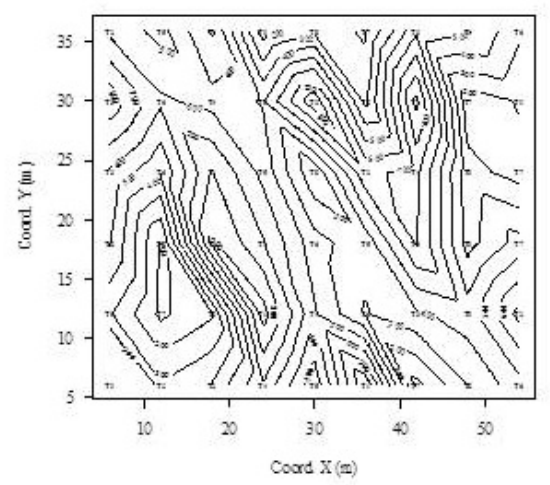

B

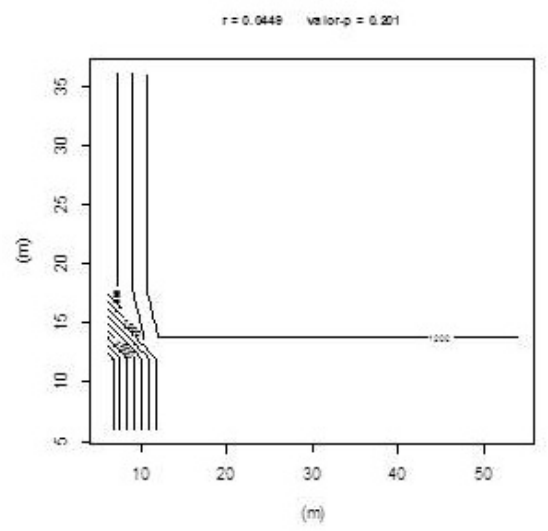

C

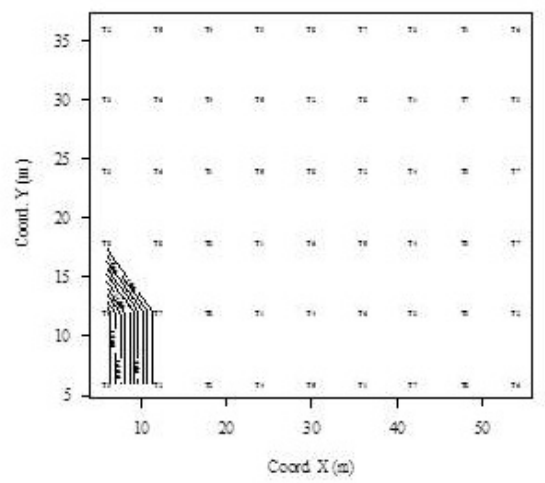

D

Figura 3. Mapas da distribuição espacial da área abaixo da curva de progresso da incidência de mofo branco (Sclerotinia sclerotiorum; AACPD) e produtividade $(\mathrm{Kg} / \mathrm{ha})$ na área experimental $\left(\mathrm{m}^{2}\right)$, A- AACPD sem efeito dos tratamentos aéreos, B- AACPD com efeito dos tratamentos aéreos, C- produtividade do feijoeiro sem efeito dos tratamentos e D- produtividade do feijoeiro com efeito dos tratamentos.

R5+10dias; V4 e R5).

A incidência do mofo-branco é uma medida baseada na porcentagem de plantas com sinais do patógeno ou sintomas da doença. No experimento a área de cultivo do feijoeiro comum estabeleceu diferentes dinâmicas do desenvolvimento do inóculo. Aos 39 DAP 1476 $\mathrm{m}^{2}$ não apresentavam incidência do mofo-branco, totalizando 75,9\% da área, enquanto $468 \mathrm{~m}^{2}$ (24,1\% da área infectada) se encontravam com incidência de até $5 \%$. Aos 46 DAP a incidência não houve expansão da área de incidência do mofo-branco. A amplitude de incidência da doença aos 53 DAP apresentou $36 \mathrm{~m}^{2}$ de área de plantio de feijão sem a incidência de mofo-branco. A amplitude da incidência do mofo-branco aos 60 DAP foi de $36 \mathrm{~m}^{2}$. Aos 67 DAP não existiu área $\left(0 \mathrm{~m}^{2}\right)$ com incidência do mofo-branco tratado. $\mathrm{O}$ cultivo que ocorreu no período de seca, ocorreu uma restrição hídrica da água fornecida em regime de pivô central, durante a condução do experimento, influenciando a incidência da doença. Numa área de $1944 \mathrm{~m}^{2}$ a epidemia foi observada, e com a evolução do ciclo da cultura houve aumento da produção de inóculo, e consequente maior quantidade de tecido lesionado, provocando aumento do número de plantas apresentando sintomas, com exceção da última avaliação, comprovando a influência da água de irrigação juntamente com o inóculo natural distribuído na área avaliada (Tab. 1). A redução da disponibilidade de água para a planta e favorabilidade deste a $S$. sclerotiorum desencadeou influência na área livre de expressão dos sintomas ( $0 \%$ de incidência), mas não na lavoura, no entanto, aos 74 DAP apresentou as maiores áreas de diferentes amplitudes de incidência do mofo-branco do feijoeiro $\left(0-5 \%-432 \mathrm{~m}^{2}, 5-10 \%-504 \mathrm{~m}^{2}, 10-15\right.$ $\%-468 \mathrm{~m}^{2}$ e $\left.14-20 \%-324 \mathrm{~m}^{2}\right)$.

Em amplitudes superiores (5-20 \%) de incidência do mofo-branco em feijoeiro, foi observada ocupação do inóculo na expressão de sintomas somente aos 53 DAP. Na amplitude máxima avaliada de 15$30 \%$ de incidência do mofo-branco, a área de ocupação do patógeno foi de $108 \mathrm{~m}^{2}, 180 \mathrm{~m}^{2}, 288$ e $324 \mathrm{~m}^{2}$, respectivamente para os dias 53 DAP, 60 DAP, 67 DAP, 74 DAP (Tab. 1).

Nas regiões com altitudes superiores a 800 metros, onde as temperaturas noturnas são amenas e sob condições de alta umidade, tem sido comum observar lavouras de soja com incidência da doença superior a $50 \%$, ou seja, a cada 100 plantas avaliadas, 50 apresentam sintomas do mofo branco (Tab. 1). Em áreas experimentais, naturalmente infestadas, tem-se observado incidência da doença acima de $70 \%$ e redução de produtividade acima de $30 \%$ (9).

A maior área de incidência do mofo-branco aos 60 DAP foi de 576 $\mathrm{m}^{2}$, mesmo submetido a diferentes tratamentos de controle, variando de $10-15 \%$ da incidência, sendo que o esperado maior incidência e ocupação aos 74 DAP, que representa fenologicamente o momento de maturação e maior janela de suscetibilidade a doença (Tab. 1), provavelmente este evento ocorreu devido os tratamentos de controle aplicados na área. Outra principal causa desse comportamento foi o descontrole na aplicação de água pelo regime de pivô central. Este fato, foi confirmado pelo teste de Mantel, que em todos os dias de avaliação não existiu diferença significativa da incidência do mofobranco nos diferentes dias de avaliação com a área geográfica $\mathrm{m}^{2}$. 
Tabela 1. Amplitudes das áreas de incidência do mofo branco do feijoeiro e a área em m2 e sua porcentagem aos 39, 46, 53, 60, 67 e 74 DAP.

\begin{tabular}{|c|c|c|c|c|c|c|c|c|c|c|c|c|}
\hline \multirow{2}{*}{$\begin{array}{l}\text { Amplitude } \\
\text { da } \\
\text { Incidência } \\
\text { do mofo- } \\
\text { branco } \\
(\%)\end{array}$} & \multicolumn{2}{|c|}{39 DAP } & \multicolumn{2}{|c|}{46 DAP } & \multicolumn{2}{|c|}{53 DAP } & \multicolumn{2}{|c|}{60 DAP } & \multicolumn{2}{|c|}{67 DAP } & \multicolumn{2}{|r|}{74 DAP } \\
\hline & $\begin{array}{c}\text { Área de } \\
\text { incidência } \\
\qquad\left(\mathbf{m}^{2}\right)\end{array}$ & $\begin{array}{c}\text { \% de } \\
\text { área } \\
\text { infectado }\end{array}$ & $\begin{array}{c}\text { Área de } \\
\text { incidência } \\
\qquad\left(\mathbf{m}^{2}\right)\end{array}$ & $\begin{array}{c}\text { \% de } \\
\text { área } \\
\text { infectada }\end{array}$ & $\begin{array}{c}\text { Área de } \\
\text { incidência } \\
\qquad\left(\mathbf{m}^{2}\right)\end{array}$ & $\begin{array}{c}\text { \% de } \\
\text { área } \\
\text { infectada }\end{array}$ & $\begin{array}{c}\text { Área de } \\
\text { incidência } \\
\qquad\left(\mathbf{m}^{2}\right)\end{array}$ & $\begin{array}{c}\text { \% de } \\
\text { área } \\
\text { infectada }\end{array}$ & $\begin{array}{c}\text { Área de } \\
\text { incidência } \\
\qquad\left(\mathbf{m}^{2}\right)\end{array}$ & $\begin{array}{c}\% \text { de } \\
\text { área } \\
\text { infectada }\end{array}$ & $\begin{array}{c}\text { Área de } \\
\text { incidência } \\
\qquad\left(\mathbf{m}^{2}\right)\end{array}$ & $\begin{array}{c}\text { \% de } \\
\text { área } \\
\text { infectada }\end{array}$ \\
\hline 0 & 1476 & 75,92 & 1476 & 75,92 & 36 & 1,85 & 36 & 1,85 & 0 & 0 & 108 & 5,5 \\
\hline $5-10$ & 0 & 0 & 0 & 0 & 324 & 16,6 & 216 & 11,1 & 252 & 12,96 & 504 & 25,9 \\
\hline $10-15$ & 0 & 0 & 0 & 0 & 288 & 14,81 & 576 & 29,6 & 234 & 16,6 & 468 & 24,07 \\
\hline $15-20$ & 0 & 0 & 0 & 0 & 108 & 5,5 & 180 & 9,2 & 288 & 14,81 & 324 & 16,6 \\
\hline Soma & 1944 & 99,99 & 1944 & 99,99 & 1080 & 55,36 & 1116 & 57,25 & 900 & 46,22 & 1836 & 94,27 \\
\hline
\end{tabular}

Tabela 2. Amplitudes das áreas abaixo da curva de progresso da incidência do mofo-branco (AACPD), ocupação ( $\left.\mathrm{m}^{2}\right)$ na área experimental, porcentagem do espaço experimental apresentando amplitude apontada (\%AI), amplitudes de produtividade ( $\mathrm{kg} / \mathrm{ha})$, área de produtividade ( $\left.\mathrm{m}^{2}\right)$ e \% do espaço experimental que apresenta a amplitude de produtividade apontada (\%EE) e seus parâmetros apontados pelo teste de Mantel.

\begin{tabular}{|c|c|c|c|c|c|}
\hline Amplitude da AACPD & $\begin{array}{c}\text { Ocupação de espaço pela } \\
\text { AACPD }\left(\mathrm{m}^{2}\right)\end{array}$ & $\%$ AI & $\begin{array}{c}\text { Amplitude de } \\
\text { produtividade (kg/ha) }\end{array}$ & $\begin{array}{c}\text { Ocupação do espaço pelo } \\
\text { produtividade }\left(\mathrm{m}^{2}\right)\end{array}$ & $\%$ EE \\
\hline $0-100$ & 108 & 5,6 & $0-1100$ & 1872 & 96 \\
\hline $200-300$ & 288 & 14,8 & $1600-2100$ & 0 & 0 \\
\hline $300-400$ & 252 & 13,0 & $2100-2600$ & 72 & 4 \\
\hline $500-600$ & 288 & 14,8 & $3100-3600$ & 0 & 0 \\
\hline $600-700$ & 108 & 5,6 & - & - & - \\
\hline $700-800$ & 180 & 9,3 & - & - & - \\
\hline \multirow{2}{*}{ Teste de Mantel } & Correlação & p-valor & & Correlação & p-valor \\
\hline & $-0,03988955$ & 0,821 & & $-0,0306541$ & 0,001 \\
\hline
\end{tabular}

Os valores de correlação foram baixos nos diferentes dias de avaliação.

A AACPD que representou e quantificou a epidemia de mofobranco no campo, representou quantidades variáveis da doença, sendo observada maior ocupação $(14,8 \%+13,0 \%+14,8 \%+14,8 \% ; 57,4 \%$ da área apresentou AACPD na amplitude de 200-600) nas amplitudes de 200 a 600 de AACPD (Tab. 2). O p-valor de Mantel apontou que rejeitou a hipótese de que a distribuição da doença na AACPD seja explicada pela área geográfica em $\mathrm{m}^{2}$, ou seja, não houve dependência espaço-temporal da AACPD com a produtividade $\left(\mathrm{Kg} \mathrm{ha}^{-1}\right)$.

No experimento a maior área de ocupação apresentou produtividade na amplitude de $0-1100 \mathrm{~kg} \mathrm{ha}^{-1}$, representando $96 \%$ da área. O teste de Mantel explicou que a ocupação geográfica apresentou diferentes patamares de produtividade do feijoeiro (Tab. 2) influenciado pela incidência do mofo branco e dos tratamentos de controle aplicados.

$\mathrm{Na}$ área estudada, observou-se grande quantidade de inóculo do fungo distribuído em padrões agregados e em elevada quantidade não existe incidência da doença no campo, sendo isto influenciado diretamente e exclusivamente pelos tratamentos.

O pico da distribuição espacial do mofo branco e a maior incidência da doença ocorreram nos dias mais próximos a colheita, não havendo dependência espaço temporal com a produtividade.

O número de focos ampliou-se e houve confluência de áreas com a presença do patógeno. 


\section{REFERÊNCIAS}

1. Almeida, A. M. R.; Ferreira, L. P.; Yorinori, J. T.; Silva, J. E. V.; Henning, A. A. Doenças da soja. Manual de fitopatologia: doenças de plantas cultivadas. V. 2. Agronômica Ceres, São Paulo. 1995 - 1997.

2. Barbosa, F. R.; Gonzaga, A. C. O. (Ed.) Informações técnicas para o cultivo do feijoeiro-comum na Região Central Brasileira: 2012 - 2014. Embrapa Arroz e Feijão, Santo Antônio de Goiás, GO, 2012.

3. Boland G. J., Hall, R. Relationships between the spatial pattern and number of apothecia of Sclerotinia sclerotiorum and stem rot of soybean. Plant Pathology, v. 37, p. 329-36, 1988.

4. Chen, R. S.; McDonald, B. A. Sexual reproduction plays a major role in the genetic structure of populations of the fungus Mycosphaerella graminicola. Genetics, Austin, v. 142, n. 4, p. 1119-1127, 1996.

5. CONAB - Companhia Nacional de Abastecimento. Acompanhamento da Safra Brasileira de Grãos 2014/2015: Monitoramento Agrícola Cultivos de verão, $2^{\mathrm{a}}$ safra e de inverno - Safra 2014/15 Brasília: Conab, 2015.

6. Ghini, R.; Hamada, E.; Bettiol, W. Impactos das mudanças climáticas sobre doenças de importantes culturas no Brasil. Embrapa meio ambiente, Jaguariúna, 2011. 356 p.

7. Görgen, C. A. Manejo do mofo-branco da soja com palhada de Brachiaria ruziziensis e Trichoderma harzianum ' 1306 '. Dissertação (Mestrado em Agronomia), Universidade Federal de Goiás, campus de Jataí, 2010, $72 \mathrm{p}$.

8. Mantel, N. The detection of disease clustering and a generalized regresf sion approach. Cancer Research, 27, 209-220; 1967.
9. Meyer, M. C.; Campos, H. D. 2009. Guerra ao mofo. Revista Cultivar. Disponível em: <http://ainfo.cnptia.embrapa.br/digital/bitstream/ item/70163/1/ID-29219.pdf>. Acesso em: 10 jan. 2016.

10. Ricardo, T. R.; Wander, A. E.; Lobo Júnior, M. 2008. Custos associados ao mofo branco (Sclerotinia sclerotiorum) em feijoeiro comum de $3^{\mathrm{a}}$ safra em Goiás. Instituto Agronômico de Campinas. Campinas, 2008. Disponível em:<ainfo.cnptia.embrapa.br/digital/bitstream/item/74992/1/pl-2008.199. pdf $>$. Acesso em: 15 ago. 2015.

11. Sartorato, A.; Rava, C. A.; Faria, J. C. 2003. Cultivo do feijoeiro comum: Doenças e métodos de controle. Embrapa arroz e feijão, Sistemas de produção 2 (Versão eletrônica). Disponível em: <sistemasdeproducao. cnptia.embrapa.br/FontesHTML/Feijao/CultivodoFeijoeiro/doenças. htm>. Acesso em: 15 ago. 2015.

12. Silva, A. R. Biotools: Tools for Biometry and Applied Statistics in Agricultural Science, Disponível em: $<$ http://CRAN.R-project.org/ package=biotools $>$, R package version 2.2, 2015 .

13. Suzui, T.; Kobayashi, T. Dispersal of ascopores of Sclerotinia sclerotiorum (Lib.) de Bary on kidney bean plants. Part I. Dispersal of ascospores from a point source of apothecia. Hokkaido National Agricultural Experiment Station Research Bulletin, v. 101, p. 137-151, 1972.

14. Talamini, V.; Lima, N. S.; Menezes, M. S.; Silva, A. M. F.; Sousa, R. C.; Silva, L. M. Qualidade fisiológica e sanitária de sementes de feijão (Phaseolus vulgaris $L$.) produzidas por agricultores familiares em Sergipe. Embrapa tabuleiros costeiros. Aracaju, 2010. 22 p. Disponível em: <http://www. cpatc.embrapa.br/publicacoes_2010/bp_62.pdf $>$. Acesso em: 15 ago. 2015

15. Williams, J. R.; Stelfox, D. Influence of farming practices in Alberta on germination and apothecium production of sclerotia of Sclerotinia sclerotiorum Canadian Journal of Plant Pathology, v. 2, p. 169-172, 1980 